Published in final edited form as:

Int J Radiat Oncol Biol Phys. 2011 November 15; 81(4): . doi:10.1016/j.ijrobp.2010.07.010.

\title{
REPEAT GAMMA KNIFE RADIOSURGERY FOR TRIGEMINAL NEURALGIA
}

\author{
Adam C. Aubuchon, B.A. ${ }^{\star}$, Michael D. Chan, M.D. ${ }^{*}$ James F. Lovato, M.S. ${ }^{\dagger}$, Christopher J. \\ Balamucki, M.D. $\ddagger$, Thomas L. Ellis, M.D.§, Stephen B. Tatter, M.D., Ph.D.§, Kevin P. \\ McMullen, M.D.., Michael T. Munley, Ph.D. ${ }^{*}$, Allan F. Deguzman, Ph.D.., Kenneth E. \\ Ekstrand, Ph.D. , J. Daniel Bourland, Ph.D. ${ }^{*}$, and Edward G. Shaw, M.D. \\ "Department of Radiation Oncology, Wake Forest University School of Medicine, Winston-Salem, \\ NC
}

tDepartment of Public Health Sciences, Wake Forest University School of Medicine, WinstonSalem, NC

§Department of Neurosurgery, Wake Forest University School of Medicine, Winston-Salem, NC ‡Department of Radiation Oncology, University of Florida, Gainesville, FL

\begin{abstract}
Purpose-Repeat gamma knife stereotactic radiosurgery (GKRS) for recurrent or persistent trigeminal neuralgia induces an additional response but at the expense of an increased incidence of facial numbness. The present series summarized the results of a repeat treatment series at Wake Forest University Baptist Medical Center, including a multivariate analysis of the data to identify the prognostic factors for treatment success and toxicity.
\end{abstract}

Methods and Materials-Between January 1999 and December 2007, 37 patients underwent a second GKRS application because of treatment failure after a first GKRS treatment. The mean initial dose in the series was $87.3 \mathrm{~Gy}$ (range, 80-90). The mean retreatment dose was $84.4 \mathrm{~Gy}$ (range, 60-90). The dosimetric variables recorded included the dorsal root entry zone dose, pons surface dose, and dose to the distal nerve.

Results-Of the 37 patients, $81 \%$ achieved a $>50 \%$ pain relief response to repeat GKRS, and $57 \%$ experienced some form of trigeminal dysfunction after repeat GKRS. Two patients (5\%) experienced clinically significant toxicity: one with bothersome numbness and one with corneal dryness requiring tarsorraphy. A dorsal root entry zone dose at repeat treatment of $>26.6 \mathrm{~Gy}$ predicted for treatment success $(61 \%$ vs. $32 \%, p=.0716)$. A cumulative dorsal root entry zone dose of $>84.3 \mathrm{~Gy}(72 \%$ vs. $44 \%, p=.091)$ and a cumulative pons surface dose of $>108.5 \mathrm{~Gy}(78 \%$ vs. $44 \%, p=.018$ ) predicted for post-GKRS numbness. The presence of any post-GKRS numbness predicted for a $>50 \%$ decrease in pain intensity (100\% vs. $60 \%, p=.0015)$.

Conclusion-Repeat GKRS is a viable treatment option for recurrent trigeminal neuralgia, although the patient assumes a greater risk of nerve dysfunction to achieve maximal pain relief.

Copyright () 2011 Elsevier Inc.

Reprint requests to: Adam C. Aubuchon, B.A., Department of Radiation Oncology, Wake Forest University School of Medicine, Medical Center Boulevard, Winston-Salem, NC 27157. Tel: (336) 407-0139; Fax: (336) 713-6565; acaubuchon@ gmail.com.

Presented, in part, in poster/abstract form at the American Society for Therapeutic Radiology and Oncology 2009 National Meeting, Chicago, IL, November 2009.

Conflict of interest: none. 


\section{Keywords}

Gamma knife; Radiosurgery; Trigeminal neuralgia; Toxicity; Repeat

\section{INTRODUCTION}

Gamma knife radiosurgery (GKRS) is an effective treatment of trigeminal neuralgia (TN). Previous series have shown the benefit to be a $250 \%$ reduction of pain in $77-95 \%$ of patients treated with GKRS (1-16). Repeat GKRS for recurrent TN has also been proved efficacious, although to date, only a limited number of small series with a combined total of 308 patients have been reported worldwide (17-25). Because of the small sample sizes, controversy has arisen regarding the optimal dose and location for repeat treatment (19-21, $23,25,26)$. Moreover, the risk factors for repeat treatment success and failure have not been consistently identified.

The goal of the present retrospective study was to report the outcomes of patients who had undergone a second GKRS for recurrent TN at Wake Forest University Baptist Medical Center. The dosimetric data were analyzed with regard to the dose, isocenter location, and isocenter distance from several important anatomic landmarks to elucidate any relationship among the dose, isocenter location, and treatment outcomes. Medical comorbidities, including multiple sclerosis, hypertension, and diabetes, were also evaluated for their correlation with treatment success or failure.

\section{METHODS AND MATERIALS}

\section{Patients}

Between January 1999 and December 2007, 652 patients underwent GKRS for medically refractory TN using the Leskell Gamma Knife (Elekta, Norcross, GA) at Wake Forest University Baptist Medical Center. The outcomes from the initial 256 patients, including 29 with recurrent $\mathrm{TN}$, were included in a previous study (27). Of the 652 patients, 54 underwent a second treatment for recurrent TN. Of the 54 patients, 37 were available for long-term follow-up, with a mean follow-up interval of 3.8 years (range, 0.6-7.2). The characteristics of these 37 patients who had undergone repeat GKRS are summarized in Table 1.

\section{Radiosurgery technique}

After a preliminary evaluation by a radiation oncologist and neurosurgeon, the patient provided informed consent for treatment with GKRS. The patient was then treated by a team consisting of a neurosurgeon, radiation oncologist, and medical physicist. The neurosurgeon applied a Leksell Model G stereotactic head frame to the patient. The patient then underwent high-resolution, noncontrast-enhanced, stereotactic magnetic resonance imaging of the brain. One patient underwent treatment planning using computed tomography. Each patient's treatment plan was generated using the Leksell GammaPlan Treatment Planning System (Elekta). A 4-mm collimator was used in all but 2 patients, for whom concentric 4and 8 -mm collimators were used. The radiation dose was prescribed to the $100 \%$ isodose line for all patients. The treatment philosophy regarding the dose and isocenter location changed during the study period owing to the evolution of treatment practice. Patients treated early in our experience had the isocenter placed more proximally such that the $50 \%$ isodose line was tangential to the pons. Later in our experience, the initial isocenter was placed more distally at the pars triangularis. The repeat treatment isocenters were generally moved distally on the nerve, unless the initial treatment isocenter had been placed distally. In such cases, the second isocenter was moved closer to the brainstem to minimize overlap. 
The mean initial prescription dose was $87.3 \mathrm{~Gy}$ (range, 80-90), with most patients receiving $90 \mathrm{~Gy}$. The collimator output factor was 0.87 for the $4-\mathrm{mm}$ collimator. The mean repeat treatment dose was $84.4 \mathrm{~Gy}$ (range, 60-90) using the same collimator output factor.

\section{Follow-up}

The patients were instructed to return for a follow-up examination 3-6 months after treatment, or sooner, if their pain had recurred. If pain control acceptable to the patient had been achieved, they were instructed to return on a need-only basis after the first posttreatment visit. Long-term follow-up data were obtained by telephone interview. The patients were excluded if they refused the telephone questionnaire or could not be interviewed by telephone. Deceased patients were included in the study if a spouse or caregiver with adequate knowledge of the patient's condition was available for questioning.

\section{Efficacy scores}

Pain relief was categorized as excellent, good, fair, or poor (EGFP). Excellent pain relief corresponded to complete pain relief without medications. Good pain relief corresponded to complete pain relief or minimal residual pain but still requiring medication. Fair pain relief corresponded to persistent symptoms, but with $>50 \%$ pain relief with or without medications. Poor pain relief corresponded to persistent symptoms with $<50 \%$ pain relief, independent of medication status. Treatment "success" was described in relationship to the outcome scores, in addition to the patient's subjective opinion determined by their satisfaction after the second GKRS.

Although recent publications on TN have more commonly used the Barrow Neurological Institute's classification for pain scores, we decided to continue using the EGFP classification to be consistent with previous publications from our institution. In addition, the EGFP classification is similar to the method described by Burchiel in a recent publication (28). According to the Burchiel classification, Grade I pain would be equivalent to an "excellent" outcome, Grade II pain would be equivalent to a "good" outcome, Grade IIIa and IIIb pain would both be included in the "fair" outcome, and Grade IV pain would be similar to a "poor" outcome in our series. One major difference between the two classifications is that $<50 \%$ pain relief, regardless of medication status, was considered a poor outcome at our institution.

We also attempted to obtain the patients' subjective pain scores using an intensity scale from 0 to 10 at diagnosis and at follow-up to help classify their pain into the EGFP categories. Subjective pain intensity values were often recorded in the patients' charts from the initial consultation notes. Missing values were obtained by telephone interview. Changes in subjective pain intensities and the outcomes determined using the EGFP classification were used as indicators of treatment efficacy.

The interval to pain relief was recorded using the variables "time to complete pain relief" and "time to partial pain relief." Pain-free intervals were recorded as partial and complete pain-free intervals, because several patients experienced recurrence with a less severe intensity than before GKRS. If a patient experienced a period of complete pain relief, in addition to a period of partial pain relief, the partial pain-free interval recorded included the complete pain-free interval. The endpoint for a partial pain-free interval was the date at which $<50 \%$ pain relief had occurred compared with the pre-treatment baseline pain level.

\section{Dosimetry}

The dosimetric variables were obtained retrospectively using the Leksell GammaPlan software and the archived treatment plans. We recorded variables that described the dose to 
critical structures and the cumulative dose between the two treatments. Such variables included the dose administered at each treatment, the cumulative dose to the trigeminal nerve, the dose to the pons surface with each treatment, the cumulative dose to the pons surface, the dose to the trigeminal ganglion with each treatment, the cumulative dose to the trigeminal ganglion, the dose to the dorsal root entry zone (DREZ) with each treatment, and the cumulative DREZ dose.

The DREZ was defined radiographically as the point at which the center of the nerve intersected the brainstem. The pons dose was defined as the closest point on the pons orthogonal to the isocenter to estimate the maximal dose at the surface of the pons. The isocenter distances were recorded from the pons and the trigeminal ganglion as variables to describe the isocenter location along the trigeminal nerve. We used the point at which the nerve crossed the petrous dura as a landmark for the distal portion of the trigeminal nerve (29). Figure 1 shows the landmarks and method on an axial magnetic resonance imaging slice captured from the GammaPlan.

Electronic medical records were used to obtain the demographic data, medical history, medications at treatment, and other relevant information. Other data were acquired from the telephone interview. Missing data are indicated by a sample size <37.

\section{Statistical analysis}

The reported $p$ values are from chi-square contingency table analyses. In addition to the previously defined pain-free intervals, the interval to pain recurrence for those who never had complete pain relief was defined as 1 day for the statistical analysis. The same method was used when defining the interval to pain recurrence from partial pain relief. We used the Kaplan-Meier method to calculate the interval-to-pain recurrence curves.

\section{RESULTS \\ Efficacy}

Of the 54 patients who had undergone repeat GKRS for recurrent TN, 37 had sufficient data to determine the outcomes. Overall, 17 patients (46\%) had excellent pain relief, 9 (24\%) had good pain relief, 5 (14\%) had fair pain relief, and $6(16 \%)$ had poor pain relief. Thus, 31 patients $(84 \%)$ experienced $>50 \%$ pain relief with repeat GKRS. The mean interval to complete pain relief was 10.5 weeks, although this interval ranged from 1 day to 78 weeks. Three patients experienced an abnormally long period to complete pain relief of $52(n=2)$ and 78 weeks. Excluding those outliers who likely experienced spontaneous remission, the mean interval to complete pain relief was 3.4 weeks. Figures 2 and 3 show the Kaplan-Meier curves for the pain-free intervals for complete and partial pain relief.

\section{Toxicity}

In the present analysis, any abnormal facial sensation, including numbness, parasthesia, and hypesthesia, was classified as treatment-related "toxicity." To determine the toxicity of repeat GKRS, the patients were asked about the presence of numbness or abnormal facial sensations after repeat GKRS. They were also questioned about any other bothersome toxicities or surgical procedures that these toxicities might have brought about. Of the 37 patients, $21(57 \%)$ had some degree of trigeminal nerve dysfunction after repeat GKRS. Of the 26 patients who had had no numbness before the second GKRS, 10 (38\%) had developed numbness after the repeat procedure. Of the 11 patients with pre-existing numbness before repeat GKRS, 3 (27\%) experienced worsening of this numbness after the second procedure. One patient with mild numbness after the first procedure experienced severe, bothersome numbness after repeat GKRS. This patient described the nerve 
dysfunction as more bothersome than the pain from TN. The patient had possibly experienced anesthesia dolorosa, although his symptoms were "bothersome numbness," not painful or burning facial numbness. One patient with worsened numbness experienced corneal dryness that required tarsorrhaphy. The patient requiring tarsorrhaphy claimed that the dryness and the subsequent procedure were preferable to the pain from TN.

An analysis was also performed to determine whether the presence of numbness correlated with the likelihood of a better or worse outcome. Of the 21 patients who had experienced some degree of numbness, $14(67 \%)$ had an excellent outcome $(n=36, p=.0057)$. In addition, 21 (100\%) of 21 patients experiencing numbness had $>50 \%$ pain relief, and 9 $(60 \%)$ of 15 patients experiencing no numbness had $>50 \%$ pain relief $(n=36, p=.0015)$. None of 6 patients with poor outcomes had sensory disturbances after GKRS.

\section{Dosimetric factors}

Of the 37 patients, 36 had received a prescription dose $>70$ Gy at repeat GKRS. The remaining patient had received $60 \mathrm{~Gy}$ in the retreatment setting. Most patients had received 80-90 Gy. An analysis was performed to determine whether a dose response was present regarding the prescription dose in the present series. No dose response was detected in our analysis.

Using the GammaPlan treatment planning system, we calculated several dosimetric variables. The results are summarized in Table 2. A statistical analysis was subsequently performed to determine whether a correlation existed between any of the calculated dosimetric variables and the treatment efficacy and toxicity. The results of the correlation of the dosimetric variables and outcomes are summarized in Table 3. The DREZ dose at repeat treatment was predictive of both efficacy and toxicity. The median DREZ dose at repeat treatment was $26.6 \mathrm{~Gy}$. Of the 19 patients receiving $\_6.6 \mathrm{~Gy}$ to the DREZ, 6 (32\%) experienced an excellent outcome. In contrast, 11 (61\%) of the 18 patients who had received $>26.6$ Gy experienced an excellent outcome $(p=.0716)$.

The most significant predictive factor of numbness in our series was the cumulative pons surface dose. The incidence of numbness was $78 \%$ in patients who had received $>108.5 \mathrm{~Gy}$ (the median pons surface dose), but it was only $39 \%$ in patients who had received less than the median dose $(p=.018)$. The DREZ dose at repeat treatment was less predictive of toxicity. Of the 19 patients receiving <26.6 Gy, 9 (44\%) experienced numbness, and $70 \%$ of the patients receiving $>26.6$ Gy experienced numbness $(p=.158)$. The cumulative DREZ dose was also predictive of the occurrence of toxicity. The median cumulative DREZ dose for the two GKRS procedures was $84.3 \mathrm{~Gy}$. Of the 18 patients receiving a total dose of $284.3 \mathrm{~Gy}, 13(72 \%)$ experienced numbness. In contrast, only 8 (44\%) of 18 patients receiving $<84.3$ Gy experienced numbness after the second GKRS $(p=.091)$.

The isocenter location along the nerve did not appear to have predictive value for our outcome measures. Neither the isocenter distance from the pons nor the isocenter distance to the petrous dura predicted for treatment efficacy or toxicity in our statistical analysis.

\section{Patient-related factors}

To determine the patient-related risk factors that predicted for treatment success or failure, we attempted to compare the efficacy and toxicity outcomes in subsets of our treatment population. Our series included 9 patients with atypical TN, as defined by Burchiel's classification. Patients with atypical TN in our series appeared to have an outcome similar to that of patients with a typical presentation. Of the patients with TN with atypical features, $44 \%$ had excellent outcomes compared with $46 \%$ of patients with typical pain and excellent 
outcomes. In addition, $77 \%$ of patients with components of atypical pain had $>50 \%$ pain relief compared with $85 \%$ of patients with typical pain. Patients with atypical pain experienced numbness in 55\% of the cases, and patients with typical pain experienced numbness in $37 \%$ of the cases. Our series included 4 patients with multiple sclerosis, 1 of whom experienced an excellent outcome. Of the remaining multiple sclerosis patients, 1 each had a good, fair, and poor outcome.

Patients with no previous surgery experienced better outcomes than those who had undergone procedures before they opted for GKRS. Of the 23 patients who had not undergone previous prior surgical intervention, $12(56 \%)$ experienced an excellent outcome compared with 4 (29\%) of the 14 patients who had undergone a previous surgery $(p=.098)$. Age, gender, pain distribution, smoking history, hypertension, current medication use, and a history of diabetes did not correlate with efficacy or toxicity.

\section{DISCUSSION}

\section{Patient outcomes}

Several institutions have reported on the use of repeat GKRS for recurrent TN as summarized in Table 4. The data for repeat GKRS for TN have been limited by the small number of patients, limited follow-up time, and heterogeneity of treatment philosophies. A total of 308 patients who had undergone repeat GKRS have been reported to date (17-25, 30 ). However, the doses used for repeat treatment have been inconsistent, ranging from 40 Gy to $>80 \mathrm{~Gy}$ in various series $(18,19)$. The target location has also varied, from the use of the same isocenter from the previous treatment to a more anterior location $(18,19,25,26)$. As a result of this heterogeneity, the published data have been difficult to interpret regarding the prognostic factors for treatment success and toxicity. Moreover, the largest series of repeat GKRS for TN had a mean follow-up time of only 15 months (20). In our retrospective analysis, the significance of the isocenter location and dose of the second treatment were analyzed. Additionally, the determination of the risk factors for the treatment response and improved quality of life were considered. Although the numbers in our series were also limited, the median follow-up of 3.8 years in our series was among the longest in published studies for such a series.

The results of the present study have demonstrated a positive correlation between patients who experienced post-therapy numbness and patients who ultimately achieved both a complete response and an improved quality of life. This correlation between toxicity and efficacy has been previously reported in patients treated with GKRS. Pollock et al. (12) described sensory disturbance in $37 \%$ of 117 patients treated with definitive GKRS at the Mayo clinic, with $12 \%$ of the patients describing clinically bothersome numbness. The Mayo group later described a greater rate of pain relief for patients treated with 90 Gy vs. 70 Gy and also noted an increased risk of trigeminal nerve dysfunction in the higher dose cohort (9). The collimator output factor used in that study was 0.80 , possibly increasing the effective dose to the nerve compared with more recent publications. Multiple series have now reported that patients who experienced sensory dysfunction were also more likely to be pain free $(30,31)$. Although the paradigm of treatment for TN with GKRS had previously been to maximize treatment efficacy while minimizing numbness, current and previous data would suggest that treating to the threshold of some clinical numbness predicts for treatment efficacy among patients with recurrent TN.

\section{Isocenter location}

The optimal location of the isocenter for the initial treatment of TN with GKRS has been somewhat controversial. Kondziolka et al. (2) targeted the proximal nerve under the 
assumption that oligodendrocytes is more sensitive to radiation than the Schwann cells and are located near the DREZ. They found an improved outcome with doses of $\geq 70 \mathrm{~Gy}$. A controlled comparison between treatment cohorts in France and Belgium showed that an increased nerve root dose correlated with greater numbness and greater pain relief (31). Although our series failed to reach statistical significance in relating the cumulative DREZ dose to a positive outcome, a trend was seen, suggesting that this relationship in the repeat treatment setting. In selected cases, delivering greater doses close to the DREZ would be difficult because the anatomic course of the nerve is in close proximity to the pons within the prepontine cistern. Restricting the dose in these cases to the surface of the pons is a challenge, requiring placement of the isocenter more distally along the nerve. Regis et al. (7) reported an excellent rate of improvement in quality of life and a low rate of toxicity with distal isocenter placement for the first GKRS. A more distally placed isocenter at the first application of GKRS in such cases would allow a future application to be delivered more proximally, where the change from oligodendrocytes to Schwann cells at the DREZ makes this portion of the nerve more radiation sensitive. If one were forced to deliver a lower dose at repeat treatment because of pontine surface dose constraints, the DREZ would be the logical location for delivery while maintaining a reasonable chance of success.

\section{Toxicity}

The toxicity seen in the present series was relatively mild and comparable to that of other studies, even considering that higher doses were used for both the first and the second treatments. One patient experienced corneal dryness, a toxicity that required temporary surgical correction. Corneal dryness has been reported in the published data to be related to the brainstem volume irradiated and is often preceded by V1 hypesthesia (32). The only case of significant toxicity in the present series was 1 patient with bothersome numbness or questionable anesthesia dolorosa, although this patient did not describe pain when completing the questionnaire. The cumulative pons surface dose was the major dosimetric factor that predicted for trigeminal dysfunction in our series. Previous published series have also reported that the brainstem dose is predictive of the development of numbness (6). Of 21 patients in the present study with trigeminal dysfunction, 20 qualitatively rated their numbness as not bothersome. One question that arises given the correlation of better quality of life with some degree of numbness is whether non-bothersome numbness should be considered an adverse event. Among surgeons who perform radiofrequency ablation as a definitive modality for TN, some degree of clinical numbness has often been considered a desired outcome. However, traditionally, in the gamma knife data for TN, numbness has been equated with toxicity. The patients included in the present series were always counseled regarding the risk of complications when undergoing a second GKRS for TN. Patients who were apprehensive about the potential toxicities were generally given a lower radiation dose at repeat treatment.

\section{Study limitations}

The present series had some limitations. First, the nature of a retrospective study is less than ideal when considering data analysis for statistical and clinical significance. During the treatment period, the repeat treatment philosophy changed on the basis of the published data. Although this allowed for different treatment strategies to be compared with each other, it limited the interpretation of the data. In addition, the patient population in our study was quite heterogeneous. Nine patients with "atypical features," in addition to 4 patients with multiple sclerosis were included in our data analysis. Multivariate analysis showed that these patients were not significantly different from the typical TN patients during the follow-up period. Historically, however, such patients have developed recurrence more quickly, and a longer follow-up period would likely show the same results in our study. An ideal study would separate typical or Type I TN from all others and provide a prospective dosimetric 
analysis. This scenario would likely require a multi-institutional study to increase the treatment population and provide more power for the study and, subsequently, greater statistical significance.

\section{CONCLUSION}

Repeat GKRS can be used to treat TN with the understanding that a second radiosurgical procedure increases the risk of significant numbness. In addition, the results of the present series demonstrated a trend toward better treatment outcomes when a higher dose to the DREZ was used at repeat treatment. The data have suggested that greater rates of numbness should be acceptable to obtain optimal pain relief. As with any surgical procedure, patients should be counseled regarding the risks and benefits of a second GKRS for TN. Specifically, patients and physicians might wish to accept more numbness in exchange for adequate pain control at repeat treatment, consistent with other ablative surgical techniques for TN.

\section{References}

1. Alpert TE, Chung CT, Mitchell LT, et al. Gamma knife surgery for trigeminal neuralgia: Improved initial response with two isocenters and increasing dose. J Neurosurg. 2005; 102(Suppl):185-188. [PubMed: 15662807]

2. Kondziolka D, Lunsford LD, Flickinger JC, et al. Stereotactic radiosurgery for trigeminal neuralgia: A multiinstitutional study using the gamma unit. J Neurosurg. 1996; 84:940-945. [PubMed: 8847587]

3. Kondziolka D, Lunsford LD, Flickinger JC. Gamma knife radiosurgery as the first surgery for trigeminal neuralgia. Stereotact Funct Neurosurg. 1998; 70(Suppl 1):187-191. [PubMed: 9782250]

4. Young RF, Vermulen S, Posewitz A. Gamma knife radiosurgery for the treatment of trigeminal neuralgia. Stereotact Funct Neurosurg. 1998; 70(Suppl 1):192-199. [PubMed: 9782251]

5. Rogers CL, Shetter AG, Fiedler JA, et al. Gamma knife radiosurgery for trigeminal neuralgia: The initial experience of the Barrow Neurological Institute. Int J Radiat Oncol Biol Phys. 2000; 47:1013-1019. [PubMed: 10863073]

6. Brisman R, Mooij R. Gamma knife radiosurgery for trigeminal neuralgia: Dose-volume histograms of the brainstem and trigeminal nerve. J Neurosurg. 2000; 93(Suppl 3):155-158. [PubMed: 11143235]

7. Regis J, Metellus P, Dufour H, et al. Long-term outcome after gamma knife surgery for secondary trigeminal neuralgia. J Neurosurg. 2001; 95:199-205. [PubMed: 11780888]

8. Brisman R. Gamma knife radiosurgery for primary management for trigeminal neuralgia. J Neurosurg. 2000; 93(Suppl 3):159-161. [PubMed: 11143236]

9. Pollock BE, Phuong LK, Foote RL, et al. High-dose trigeminal neuralgia radiosurgery associated with increased risk of trigeminal nerve dysfunction. Neurosurgery. 2001; 49:58-64. [PubMed: 11440460]

10. Zheng LG, Xu DS, Kang CS, et al. Stereotactic radiosurgery for primary trigeminal neuralgia using the Leksell Gamma unit. Stereotact Funct Neurosurg. 2001; 76:29-35. [PubMed: 12007276]

11. Maesawa S, Salame C, Flickinger JC, et al. Clinical outcomes after stereotactic radiosurgery for idiopathic trigeminal neuralgia. J Neurosurg. 2001; 94:14-20. [PubMed: 11147887]

12. Pollock BE, Phuong LK, Gorman DA, et al. Stereotactic radiosurgery for idiopathic trigeminal neuralgia. J Neurosurg. 2002; 97:347-353. [PubMed: 12186463]

13. Petit JH, Herman JM, Nagda S, et al. Radiosurgical treatment of trigeminal neuralgia: Evaluating quality of life and treatment outcomes. Int J Radiat Oncol Biol Phys. 2003; 56:1147-1153. [PubMed: 12829153]

14. Brisman R. Microvascular decompression vs. gamma knife radiosurgery for typical trigeminal neuralgia: Preliminary findings. Stereotact Funct Neurosurg. 2007; 85:94-98. [PubMed: 17167238] 
15. Fountas KN, Lee GP, Smith JR. Outcome of patients undergoing gamma knife stereotactic radiosurgery for medically refractory idiopathic trigeminal neuralgia: Medical College of Georgia's experience. Stereotact Funct Neurosurg. 2006; 84:88-96. [PubMed: 16825804]

16. Brisman R. Gamma knife surgery with a dose of 75 to 76.8 Gray for trigeminal neuralgia. J Neurosurg. 2004; 100:848-854. [PubMed: 15137604]

17. Pollock BE, Foote RL, Stafford SL, et al. Results of repeated gamma knife radiosurgery for medically unresponsive trigeminal neuralgia. J Neurosurg. 2000; 93(Suppl 3):162-164. [PubMed: 11143237]

18. Hasegawa T, Kondziolka D, Spiro R, et al. Repeat radiosurgery for refractory trigeminal neuralgia. Neurosurgery. 2002; 50:494-502. [PubMed: 11841716]

19. Shetter AG, Rogers CL, Ponce F, et al. Gamma knife radiosurgery for recurrent trigeminal neuralgia. J Neurosurg. 2002; 97:536-538. [PubMed: 12507092]

20. Brisman R. Repeat gamma knife radiosurgery for trigeminal neuralgia. Stereotact Funct Neurosurg. 2003; 81:43-49. [PubMed: 14742963]

21. Herman JM, Petit JH, Amin P, et al. Repeat gamma knife radiosurgery for refractory or recurrent trigeminal neuralgia: Treatment outcomes and quality-of-life assessment. Int J Radiat Oncol Biol Phys. 2004; 59:112-116. [PubMed: 15093906]

22. Pollock BE, Foote RL, Link MJ, et al. Repeat radiosurgery for idiopathic trigeminal neuralgia. Int $\mathbf{J}$ Radiat Oncol Biol Phys. 2005; 61:192-195. [PubMed: 15629611]

23. Zhang P, Brisman R, Choi J, et al. Where to locate the isocenter? The treatment strategy for repeat trigeminal neuralgia radiosurgery. Int J Radiat Oncol Biol Phys. 2005; 62:38-43. [PubMed: 15850900]

24. Wang L, Zhao ZW, Qin HZ, et al. Repeat gamma knife radiosurgery for recurrent or refractory trigeminal neuralgia. Neurol India. 2008; 56:36-41. [PubMed: 18310835]

25. Gellner V, Kurschel S, Kreil W, et al. Recurrent trigeminal neuralgia: Long-term outcome of repeat gamma knife radiosurgery. J Neurol Neurosurg Psychiatry. 2008; 79:1405-1407. [PubMed: 18420725]

26. Urgosik D, Liscak R, Novotny J Jr, et al. Treatment of essential trigeminal neuralgia with gamma knife surgery. J Neurosurg. 2005; 102(Suppl):29-33. [PubMed: 15662776]

27. Balamucki CJ, Stieber VW, Ellis TL, et al. Does dose rate affect efficacy? The outcomes of 256 gamma knife surgery procedures for trigeminal neuralgia and other types of facial pain as they relate to the half-life of cobalt. J Neurosurg. 2006; 105:730-735. [PubMed: 17121135]

28. Burchiel K. Trigeminal neuralgia (editorial). J Neurosurg. 2009; 111:733-736. [PubMed: 19392597]

29. Fuentes S, Delsanti C, Metellus P, et al. Brainstem metastases: Management using gamma knife radiosurgery. Neurosurgery. 2006; 58:37-42. [PubMed: 16385327]

30. Dvorak T, Finn A, Price LL, et al. Retreatment of trigeminal neuralgia with gamma knife radiosurgery: Is there an appropriate cumulative dose? J Neurosurg. 2009; 111:359-364. [PubMed: 19326978]

31. Massager N, Murata N, Tamura M, et al. Influence of nerve radiation dose in the incidence of trigeminal dysfunction after trigeminal neuralgia radiosurgery. Neurosurgery. 2007; 60:681-688. [PubMed: 17415205]

32. Naseri A, Patel NP. Vision loss as a complication of gamma knife radiosurgery for trigeminal neuralgia. Br J Ophthalmol. 2004; 88:1225-1226. [PubMed: 15317722]

33. Huang C, Chuang J, Tu H, et al. Repeated Gamma Knife Surgery for Refractory Trigeminal Neuralgia. J Neurosurg. 2006; 105(suppl):99-102. [PubMed: 18503339] 


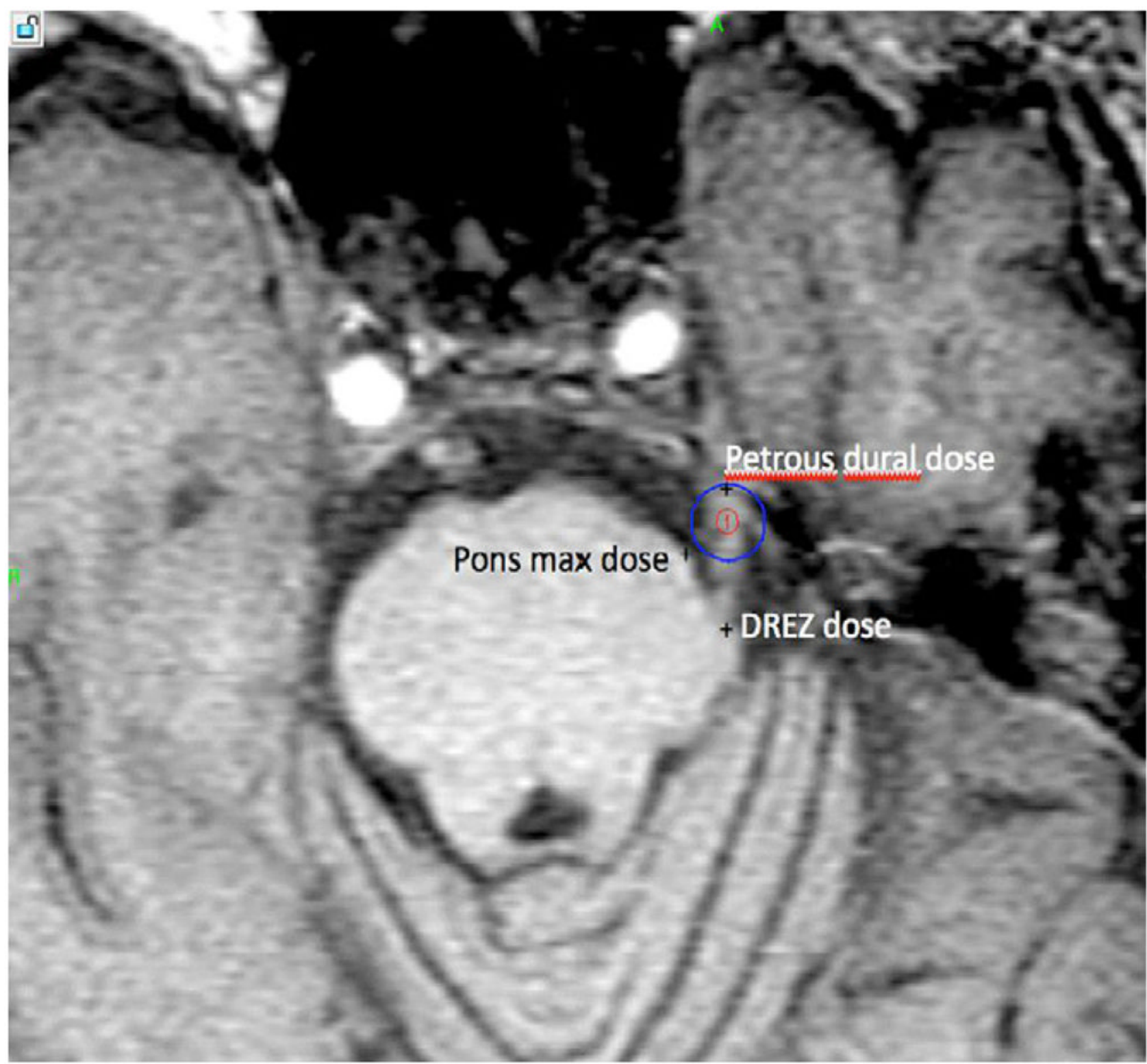

Fig. 1.

Axial slice magnetic resonance image showing 50\% and 95\% isodose curves from gamma knife radiosurgery plan using single 4-mm shot. Crosshairs placed at dorsal root entry zone to calculate point dose. "Measure" tool from GammaPlan software calculated distance from shot to pons and gasserian ganglion. 


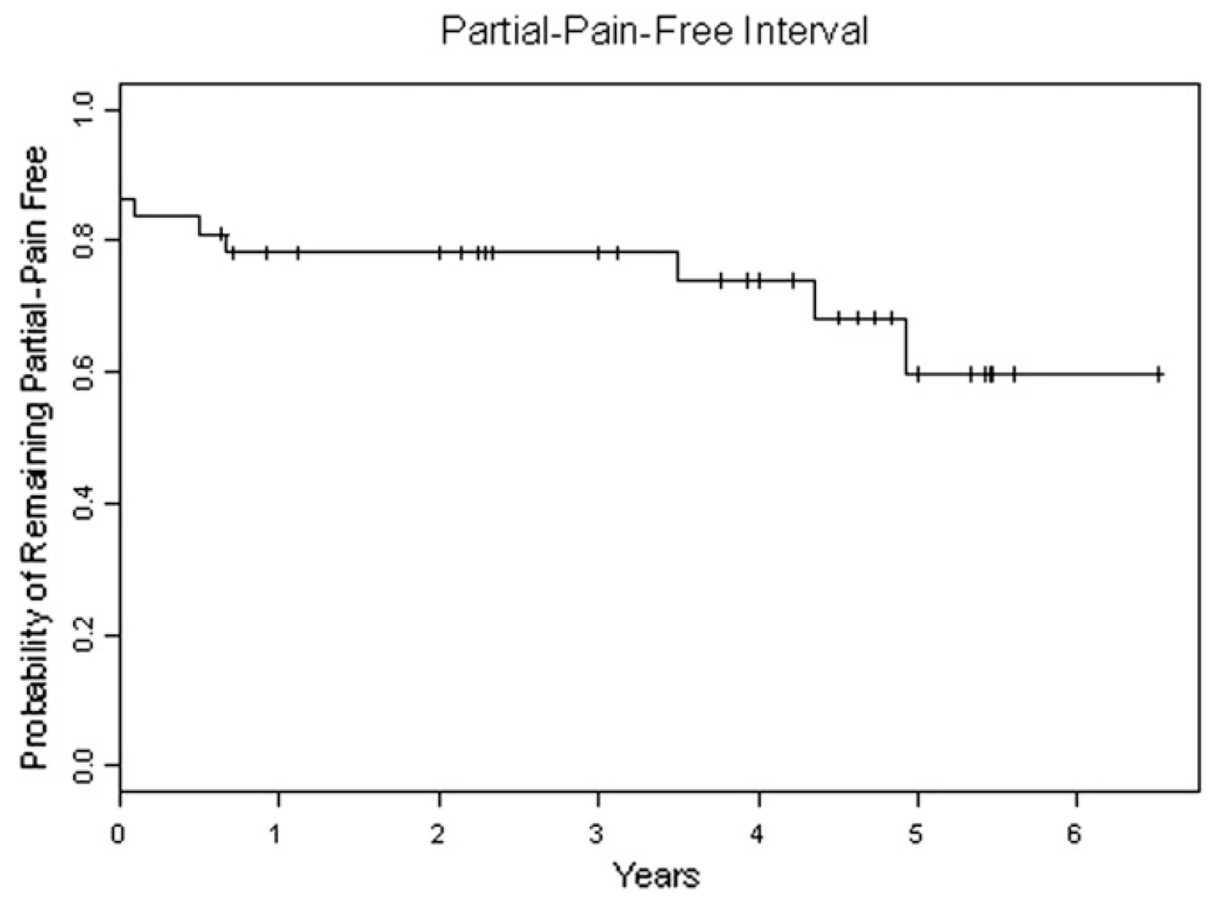

Fig. 2.

Kaplan-Meier curve showing survival time with some degree of pain relief improved from pretreatment intensity. 


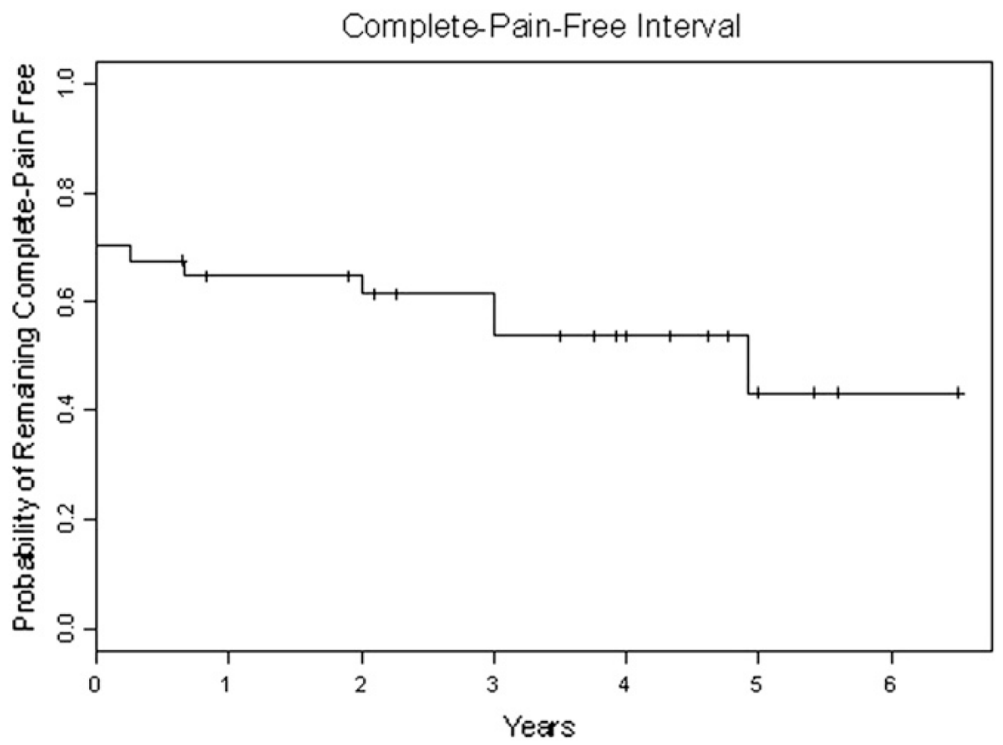

Fig. 3.

Kaplan-Meier curve showing survival time with complete pain relief. 


\section{Table 1}

Demographics

\begin{tabular}{|c|c|}
\hline Demographic & Value \\
\hline \multicolumn{2}{|l|}{ Gender } \\
\hline Male & $17(46)$ \\
\hline Female & $20(54)$ \\
\hline \multicolumn{2}{|l|}{ Pain side } \\
\hline Right & $20(54)$ \\
\hline Left & $17(46)$ \\
\hline \multicolumn{2}{|l|}{ Distribution } \\
\hline $\mathrm{V} 1$ & $2(5)$ \\
\hline $\mathrm{V} 1, \mathrm{~V} 2$ & $1(3)$ \\
\hline $\mathrm{V} 1, \mathrm{~V} 2, \mathrm{~V} 3$ & $6(16)$ \\
\hline $\mathrm{V} 2$ & $15(41)$ \\
\hline $\mathrm{V} 2, \mathrm{~V} 3$ & $9(24)$ \\
\hline V3 & $4(11)$ \\
\hline \multicolumn{2}{|l|}{ Pain type } \\
\hline Typical & $29(78)$ \\
\hline Atypical & $8(22)$ \\
\hline \multicolumn{2}{|l|}{ Comorbidities } \\
\hline Multiple sclerosis & $4(11)$ \\
\hline Hypertension & $16(43)$ \\
\hline Diabetes & $3(8)$ \\
\hline \multicolumn{2}{|l|}{ Autoimmune disease } \\
\hline Smoker & $10(27)$ \\
\hline Never smoked & $15(41)$ \\
\hline Former smoker & $12(32)$ \\
\hline Previous surgical procedures & $13(35)$ \\
\hline \multicolumn{2}{|l|}{ Surgical procedures $(n)$} \\
\hline 0 & $24(65)$ \\
\hline 1 or 2 & $9(24)$ \\
\hline 23 & $4(11)$ \\
\hline Surgical procedures between GK treatments & $2(5)$ \\
\hline \multicolumn{2}{|l|}{ Procedure before first GKRS } \\
\hline Microvascular decompression & $6(16)$ \\
\hline Rhizotomy & $10(27)$ \\
\hline Nerve block & $5(14)$ \\
\hline Mean dose at first GKRS (Gy) & 87.3 \\
\hline Mean dose at second GKRS (Gy) & 84.2 \\
\hline Mean total dose (Gy) & 171.5 \\
\hline Mean interval between GKRS (y) & 1.3 \\
\hline Mean duration of pain before second GKRS (y) & 1.7 \\
\hline
\end{tabular}

Int J Radiat Oncol Biol Phys. Author manuscript; available in PMC 2013 December 05. 
Abbreviations: $\mathrm{GK}=$ gamma knife; GKRS = gamma knife stereotactic radiosurgery.

Data presented as numbers of patients, with percentages in parentheses, unless otherwise noted. 
Table 2

Dosimetric characteristics of gamma knife stereotactic radiosurgery

\begin{tabular}{lrrr}
\hline Variable & First GKRS & Second GKRS & Cumulative \\
\hline Mean isocenter distance from pons & 2.4 & 3.2 & \\
Mean isocenter distance from petrous dura & 4.7 & 3.7 & \\
Mean pons surface dose & 46.8 & 59.1 & 105.9 \\
Mean dose of nerve at petrous dura & 29.5 & 24.4 & 43.5 \\
Mean DREZ dose & 46.3 & 34.3 & 80.6 \\
\hline
\end{tabular}

Abbreviations: GKRS = gamma knife stereotactic radiosurgery; DREZ = dorsal root entry zone. 
